\title{
Inferring Availability for Communication in Smart Homes Using Context
}

\author{
Julien Cumin*†, Grégoire Lefebvre*, Fano Ramparany*, James L. Crowley ${ }^{\dagger}$ \\ * Orange Labs \\ Meylan, France \\ \{julien1.cumin, gregoire.lefebvre, fano.ramparany\}@orange.com \\ †Univ. Grenoble Alpes, Inria, CNRS, Grenoble INP, LIG, F-38000 \\ Grenoble, France \\ james.crowley@inria.fr
}

\begin{abstract}
This paper presents a technique for inferring the availability of people to receive communications based on their current situation. This technique uses a context model that associates situations with learned preferences for communications. Situations are represented as a tuple composed of identity, time, place, activity, correspondent, and communication modality. A place-based activity recognition technique is used to recognize the current activity from sensor data. Availability for communications is learned from history of the occupant's preferences of availability for each situation. The system is demonstrated using a dataset of availability preferences recorded from the occupant of an instrumented apartment over a period of 4 weeks. Performance of the system is compared under various assumptions of independence of availability from some of the context elements. The paper is completed with a discussion of how such techniques can be used to construct an intelligent communications assistant for smart home services.
\end{abstract}

Index Terms-ambient intelligence, context-aware services, smart homes, activity recognition

\section{INTRODUCTION}

Constant advances in Internet of Things technologies in the recent years have opened up a plethora of possible new intelligent services. One such service is that of a communication assistant for managing incoming communications from outside. Such a service would act as a secretary to advise outside correspondents concerning appropriate times to initiate a communication with an occupant of the home, to suggest alternative communication modes that the occupant may prefer, or to automatically redirect a communication from one mode to another mode that is more appropriate for the current situation of the occupant.

To provide such services, a communication assistant would require information about the availability for communication of the occupant. While this information can be inferred from specific data sources in certain environments (e.g. in professional offices, the availability of an employee can be inferred from their schedule), communications availability in private homes is highly dependent on individual preferences. Estimating the availability for communication of occupants from low-level data sources that are typically used in smart home systems currently (such as presence sensors, smart plugs, door opening sensors, etc.) is thus a difficult task.
This paper reports on experiments with the use of context to estimate availability for communication. Innovations include (1) the use of a context model based on identity, time, place, activity, correspondent and communication modality, (2) the use of place-based approaches for activity recognition from sensor data, (3) the use of an availability preference database, which contains availabilities in past situations. The proposed techniques are evaluated using a new publicly available data set [1] that provides a recorded history of communication availability recorded over a period of 4 weeks in a highly instrumented apartment.

This paper is organized as follows: Section II provides a summary of related papers on estimation of availability in both home and professional environments. Section III presents a model of availability as a function of context information, including a discussion of state values for each variable in the context model. Section IV describes the experimental results. This is followed by a conclusion on the design of communication assistance systems based on availability estimation, in Section V.

\section{RELATED WORKS ON AVAILABILITY ESTIMATION}

In the literature, works on estimating the availability ${ }^{1}$ of people have been mostly focused on professional environments [2]. In [3], an availability estimation approach is proposed in the context of office work. The importance of head posture as well as computer interactions is studied in relation with the availability of employees. In [4], a system for automatic recommendation of communication modalities to use at work is proposed; modalities are suggested depending on the availability of the employee that needs to be contacted. Availability is, in that case, deduced from common rules that exist at work, such as whether the office door is opened, the schedule of the employee, the sound levels around their computer, etc. In [5], an email delivery system based on users' availability is presented. Here, availability is determined based on interactions with the computer. For example, a moment of switch between two applications is considered to be an opportune time to interrupt the user with an email. In these

\footnotetext{
1 "Interruptibility" is also a term often used instead of "availability".
} 
examples, availability is estimated based on predefined rules (e.g. an employee is probably unavailable if their office's door is closed), which is reasonable in professional environments. However, such rules do not generally exist at home, where availability is highly dependent on individual preferences.

Nevertheless, there are some papers on the subject in the literature: in [6], the availability of occupants of homes is estimated using data from cameras and microphones. The availability is deduced from audio-visual features, using a support vector regression approach. This exploits features extracted from sensor data to infer availability, as opposed to using richer context information gathered from available sensors. On the other hand, in [7], a statistical study of availability in the home shows that identity, place, and activity are all important indicators for estimating availability. They also conjecture that time plays an important role. Both of these papers present different approaches to the problem of availability estimation: in one approach, availability is estimated using machine learning, from data produced by sensors rich in information; in the second approach, availability is estimated from other context information of the home. The approach described in this paper mixes these 2 methods, estimating availability using rich information, including activity, which is itself recognized from data produced by sensors weak in information such as presence sensors, smart plugs, etc.

\section{Availability for COMmunication in Homes}

Availability for communication at home depends on context. The most widely used elements of context at home are identity, time, place and activity, reported, for example in [7] and in [8]. In addition to these contextual elements, availability for communication depends on the person seeking to communicate with the occupant (the correspondent), and the communication modality, that is the mean of communication. This suggests that a communications assistant should be able to predict availability based on the tuple (identity, time, place, activity, correspondent, modality).

Before computing the availability function of one occupant Av (as presented in Section III-A), identity, time, place and activity need to be evaluated from sensor data:

- Identity: we assume here that the identity is either given by a perfect identity detector, or is unique in the home.

- Time: sensor events are timestamped, therefore time is trivial to evaluate.

- Place: we assume in this work that the localization of sensors and activities is given. We make no assumption on the localization of the occupant; Section III-B shows why this information is not needed in our approach.

- Activity: we present our activity recognition approach in Section III-B.

Secondly, we propose an availability preferences database that can be used to compute Av for each possible (correspondent, modality) couple. This database as well as the domains of values of correspondents, modalities, and availability are presented in Section III-C.

\section{A. Availability as a function of Context}

Elements of context information are not independent. For example, place information can be used to improve activity recognition [9]. Thus, we expect to be able to use the other 6 elements of context to determine availability. More formally, we define the availability of an occupant in the domain $\mathcal{A} \mathcal{V}$ to be the image through a function Av:

$$
\begin{gathered}
\mathrm{Av}: \mathcal{I} \times \mathcal{T} \times \mathcal{P} \times \mathcal{A} \times \mathcal{C} \times \mathcal{M} \longrightarrow \mathcal{A} \mathcal{V} \\
(i, t, p, a, c, m) \longmapsto \operatorname{Av}(i, t, p, a, c, m)
\end{gathered},
$$

of the corresponding context tuple (identity, time, place, activity, correspondent, modality) in $\mathcal{I} \times \mathcal{T} \times \mathcal{P} \times \mathcal{A} \times \mathcal{C} \times \mathcal{M}$. In reality, the availability of an occupant is also dependent on their current thoughts, their mood, etc. [10], which cannot be automatically captured by a smart home system currently.

\section{B. Activity Recognition}

Our availability inference model uses a place-based technique for activity recognition from low-level sensor data described in [9]. In this approach, each place of the home is assigned a classifier, which is responsible for recognizing activities that can occur in this place, using only the sensors relevant to this place. A step of fusion combines the decisions taken in each place into a final decision about the class of the input activity instance.

Using this approach provides multiple benefits: first, it has been shown in [9] to improve recognition rates on a specific dataset (which we also observe in the experimental section of this paper on another dataset in Section IV-B); second, it is faster to train and easier to parametrize; third, its subdivision per place circumvents the need for localizing the occupant in the home, as this can deduced from the recognized instance.

\section{Database of Availability Preferences}

The database of availability preferences contains records of the availabilities of an occupant for activity instances that occurred previously in the home. For each of these instances, a value of availability is indicated for each possible (correspondent, modality) couple. The availability function Av can use this database to infer the availability of each (correspondent, modality) couple for a newly recognized activity.

Correspondents can be identified uniquely through their identity. However, this makes the number of possible correspondents very high, even though the availability of an occupant will probably not vary between similar types of correspondents. In fact, it seems more reasonable to set the domain of correspondents to a set of categories of people known to the occupant. We have identified the following categories (which are similar to the ones identified in [11]) for which the occupant will choose significantly different patterns of availability:

- close relatives

- distant relatives

- friends

- strangers
- professional colleagues

- professional supervisors

- acquaintances 
The number of communication modalities available in the home has greatly increased over the last decade, so much so that listing all those modalities is hardly possible. Nevertheless, we group those modalities into hierarchical categories: first, we separate them between synchronous (e.g. a phone call) and asynchronous (e.g. an email) modalities; second, we separate them based on the type of data exchanged to communicate (voices, videos, or text); last, we divide them based on the actual type of device used. This leads us to the following hierarchical domain of values for modality:

$$
\begin{array}{cc}
\text { - Synchronous } & \text { - Asynchronous } \\
\text { - Voice } & - \text { Voice } \\
* \text { Landline phone } & * \text { Landline phone } \\
* \text { Mobile phone } & * \text { Mobile phone } \\
* \text { Computer } & - \text { Text } \\
\text { - Video } & * \text { Mobile phone } \\
* \text { Mobile phone } & * \text { Computer } \\
* \text { Computer } & \\
\text { - Text } & \\
* \text { Mobile phone } & \\
* \text { Computer } &
\end{array}
$$

In the literature, there seems to be no consensus on the domain of values to use to represent availability. For example, a 4-point scale (highly available, available, unavailable, highly unavailable) is used in [4], and a 5-point scale (from highly available to highly unavailable) is used in a later related work [12], and no motivation seems to be given for this change nor for the original choice of the 4-point scale. A 3-point scale (high, medium, low) is used in [5]. A 5 point scale with relative values (from least interruptible to most interruptible) is used in [6]. A survey on interruptibility prediction reports the same observation: both ordered sets of various gradations, as well as qualitative sets, are used to represent availability, with no established consensus or clear justifications for those choices [2]. As such, we decided to use a set of symmetric gradations to label availability:

- -2 : definitely unavailable;

- -1 : preferably unavailable;

- 0: no opinion, does not know;

\begin{tabular}{|c|c|c|c|c|c|c|c|c|c|c|c|}
\hline \multirow[b]{3}{*}{ Correspondent } & \multicolumn{7}{|c|}{ Synchronous } & \multicolumn{4}{|c|}{ Asynchronous } \\
\hline & \multicolumn{3}{|c|}{ Voice } & \multicolumn{2}{|c|}{ Video } & \multicolumn{2}{|c|}{ Text } & \multicolumn{2}{|c|}{ Voice } & \multicolumn{2}{|c|}{ Text } \\
\hline & $\mathrm{L}$ & M & $\mathrm{C}$ & $\mathrm{M}$ & $\mathrm{C}$ & M & $\mathrm{C}$ & $\mathrm{L}$ & M & $\mathrm{M}$ & $\mathrm{C}$ \\
\hline Close Relatives & -1 & 1 & -2 & -2 & -2 & 2 & -2 & 0 & 0 & 2 & 2 \\
\hline Distant Relatives & -1 & 1 & -2 & -2 & -2 & 2 & -2 & 0 & 0 & 1 & 2 \\
\hline Prof. colleagues & -2 & -2 & -2 & -2 & -2 & -2 & -2 & -2 & -2 & -2 & 2 \\
\hline Prof. supervisors & -2 & -2 & -2 & -2 & -2 & -2 & -2 & -2 & -2 & -2 & 2 \\
\hline Friends & -1 & 1 & -2 & -2 & -2 & 2 & -2 & 0 & 0 & 1 & 2 \\
\hline Acquaintances & -1 & 1 & -2 & -2 & -2 & 1 & -2 & 0 & 0 & -1 & 2 \\
\hline Strangers & -1 & 1 & -2 & -2 & -2 & 1 & -2 & 0 & 0 & -1 & 2 \\
\hline
\end{tabular}

- 1: preferably available;

- 2: definitely available.

This scale allows the occupant to choose their availability both as a hard decision (definitely unavailable/available), as well as a preference (preferably unavailable/available). It also allows the occupant to be neutral about their availability, which seems important from a user-centric standpoint, considering that evaluating one's own availability is often difficult. The symmetry of this scale makes it easier for the occupant to select their availability, by comparing their current situation to the availability of similar or of completely opposite situations.

The availability preferences database contains, for each of its recorded activity instances, the availability values from -2 to 2 for each of the possible 77 (correspondent, modality) couples.
TABLE I

Preset AVAILABILITIES FOR WATChing TV IN THE Living Room.

\section{EXPERIMENTAL RESULTS}

\section{A. The Orange4Home Dataset}

Orange 4 Home $e^{2}[1]$ is a dataset of routine activities collected in a fully instrumented apartment. Data from 236 heterogeneous sensors were collected over a period of 4 consecutive weeks of working days; 493 instances of activities were labelled in situ by the occupant during that period, following a predefined realistic routine of living; those instances are each labelled as one of 27 high-level classes (such as Cooking or Watching TV) that can occur in 8 different places of the home.

The Orange4Home dataset also contains data about the availability levels of the occupant (although this was not reported in [1]). For each activity instance, the occupant had to set their availability in situ. Since the number of couples is high (77, as presented in Section III-C), and availability is often similar between activities of the same class, those availability values were actually preset by the occupant before the experiment. An example of such preset for the activity class Watching TV in the Living Room is shown in Table I. Then, for each activity instance, the occupant only changed the availability of the few couples for which they deemed the preset availability value to not fit their current situation. For example, only 2 changes occurred for Watching $T V$ in the Living room, while 24 changes occurred for Computing in the Office) during the 4 weeks of experiment.

\section{B. Place-Based Activity Recognition}

Activity recognition was performed after the following preprocesses: a low-pass filter was applied on the data, and data was standardized to have a mean of 0 and standard deviation 1 for each sensor. Data were then resampled so that each activity instance is 20 samples long; those samples are concatenated and used as feature vectors to the classifiers. The first two weeks of data were used as a training set, and the third week as a validation set for training classifiers. Data from the fourth week were used as the test set.

As in [9], we compare in Table II the F1 scores of a Home configuration, where all sensors are used and all activities can be decided by a single classifier, to the results of the placebased approach where 8 different classifiers (one for each place) are combined using a decision fusion step. The decision

\footnotetext{
${ }^{2}$ https://amiqual4home.inria.fr/orange4home/
} 
TABLE II

F1 Scores of the Home Configuration and of the Place-Based APPROACH, FOR TWO DIFFERENT TYPES OF CLASSIFIERS

\begin{tabular}{ccc}
\hline & \multicolumn{2}{c}{ Classifier } \\
\cline { 2 - 3 } Approach & MLP & SVM \\
\hline Place-based & $93.05 \% 1$ & $92.08 \%^{2}$ \\
Home & $77.85 \%$ & $89.61 \%$ \\
\hline \multicolumn{2}{c}{ Decision Fusion algorithm used: } \\
\multicolumn{2}{c}{ Dempster-Shafer fusion } \\
\multicolumn{2}{c}{ 2 Majority vote fusion } \\
\hline
\end{tabular}

fusion algorithms tested in this experiment are presented in [13]. The parameters of the Home classifiers as well as the place-based classifiers were optimized on the validation set using a grid-search approach. We chose here the MultiLayer Perceptron (MLP) and the Support Vector Machine (SVM) to obtain baseline results; we used the implementations of the Weka library [14] for those two classifiers.

We observe in Table II that the place-based approach significantly outperforms the Home approach on this dataset, for both the MLP (93.05\% compared to $77.85 \%$ ) and the SVM $(92.08 \%$ compared to $89.61 \%)$. The gap between the two approaches is significantly larger than the one reported in [9] on another dataset: this can be explained from the fact the dataset used in this study contained a large number of body-worn sensors as data sources, which lowers the benefits of the place-based approach. Indeed, since body-worn sensors are used as data sources in all places and since any activity will have an impact on those sensors, the classifiers of all places will observe this data and tend to wrongly assume that something is happening in their respective place.

\section{Inferring Availability from Activity Recognition}

Mistaking availability for unavailability (and vice versa) is a significant error, as the services offered by a communication assistance system will surely be different between those two cases. On the other hand, confusing definite availability and preferable availability (and similarly for unavailability) is less damaging, as the occupant is still available (or unavailable) in both cases. In numerical terms, this means that large absolute error values are worse than small absolute error values. Similarly, errors of availability inference made on long activities are worse than those made on short ones. We thus use a Duration-Weighted Root Mean Squared Error (DWRMSE) to evaluate the results of availability inference, as this metric penalizes more both large absolute errors (due to the quadratic term) and errors made on long activities (due to the weight). For a test set of $n$ activity instances, where the inferred availability is $\hat{A v}$ and the true availability is $A v$, we have:

$$
\text { DWRMSE }=\sqrt{\sum_{i=1}^{n} w_{i}\left(\hat{\mathrm{Av}}_{i}-\mathrm{Av}_{i}\right)^{2}}, w_{i}=\frac{d_{i}}{\sum_{j=1}^{n} d_{j}}
$$

where $d_{i}$ is the duration of activity $i$. Note that the standard RMSE is a special case of DWRMSE when $\forall i, w_{i}=\frac{1}{n}$.
TABLE III

DWRMSE AND ERROR RATE OF AVAILABILITY INFERENCE AVERAGED BY CORRESPONDENT, BY MODALITY, AND IN TOTAL.

\begin{tabular}{llcc}
\hline & & DWRMSE & Error rate \\
\hline Correspondent & Close relatives & 0.126 & 0.019 \\
& Distant relatives & 0.119 & 0.019 \\
& Prof. colleagues & 0.222 & 0.002 \\
& Prof. supervisors & 0.147 & 0.006 \\
& Friends & 0.128 & 0.020 \\
& Acquaintances & 0.489 & 0.023 \\
Strangers & 0.137 & 0.019 \\
\hline Modality & Sync. Voice landline & 0.067 & 0.007 \\
& Sync. voice mobile & 0.133 & 0.007 \\
& Sync. voice computer & 0 & 0 \\
& Sync. video mobile & 0.220 & 0.003 \\
& Sync. video computer & 0.220 & 0.003 \\
& Sync. text mobile & 0 & 0 \\
& Sync. text computer & 0 & 0 \\
Async. voice landline & 0.152 & 0.037 \\
Async. voice mobile & 0.180 & 0.048 \\
& Async. text mobile & 0.521 & 0.044 \\
Async. text computer & 0.380 & 0.022 \\
\hline
\end{tabular}

We will also use the error rate as an evaluation metric, that is, the number of inferred availabilities that are not equal to their corresponding true availabilities, divided by the total number of inferred availabilities. The error rate will indicate how often availability is incorrectly inferred, and DWRMSE will indicate how bad those incorrect inferences are.

Following an activity recognition step, we can now infer availability for each activity instance. We use the place-based MLP activity recognition approach to provide the activity labels (as this approach is the most accurate one with an F1 score of $93.05 \%$ ). We use a simple baseline inferring approach as the Av function described in Section III-A: the availability of an activity instance from activity class $a$, for a couple of correspondent and modality $(c, m)$, is the average (rounded to the closest integer) of all availabilities for the couple $(c, m)$ of instances of $a$ in the training set.

Table III reports the DWRMSE and error rates of this availability inference approach, averaged for each category of correspondent, for each category of modality, and averaged in total over all activity instances. We see that the average total error rate is fairly small (0.015): since there are 108 instances of activities during the test week, and since each activity instance accounts for 77 availability values (one for each couple of (correspondent, modality)), there are in total 128 badly inferred availability values, over the total 8316 availability values. Given the simple averaging approach that was used, this implies that the occupant has rarely changed their level of availability from the preset values that they had chosen before the experiment. Assuming that this behaviour is common to most occupants, inferring availability is thus only difficult in the rare cases where the occupant's availability deviates significantly from their preferences.

We can observe that the availability for correspondents and modalities are not all equivalently well inferred: in particular, 
we see that professional colleagues $(0.222)$, professional supervisors (0.147), acquaintances (0.489) and strangers (0.137) have higher DWRMSE than the other 3 groups of correspondents. This may be explained by the fact that those categories of correspondents are the most emotionally distant from the occupant, and their availability is thus much less clear to the occupant and depends on the actual content of the conversation that the occupant is expecting.

Availability for synchronous modalities is often dependent on physical restrictions. For example, it is physically impossible to stay on the phone with someone while taking a shower. Thus, many availability values for such modalities are implicitly "imposed" by their own nature, which reflects in the preset values of the occupant and thus in the smaller DWRMSE values observed. Such restrictions obviously do not exist for asynchronous modalities, and are therefore more freely set by the occupant, thus leading to higher inference errors. This is illustrated by the high DWRMSE (0.220) of the two synchronous video modalities.

\section{Independence of Availability from Correspondents, Modal- ities, or Activities}

The assumption that availability is dependent on correspondents, modalities, as well as activities, implies that there is a significant number of different availability values for the occupant to both preset and choose from (when changing the preset values for a particular activity instance). For instance, with 20 activity classes, 7 groups of correspondents and 11 groups of modalities, there are 1540 specific availability values. The higher this number is, the less likely a smart home occupant will be to willingly provide their availability for each of those possibilities. Therefore, it is interesting to see whether availability inference is greatly worsened or not under one of the following assumptions:

A1) availability is independent of the correspondent;

A2) availability is independent of the modality;

A3) availability is independent of both the correspondent and the modality;

A4) availability is dependent on the place in which the activity takes place, but not the activity itself.

Each of those assumptions lead to the following availability inference approach: the availability of an activity instance from activity class $a$, for a couple of correspondent and modality $(c, m)$, is the average (rounded to the closest integer) of:

1) all availabilities for couples with modality $m$ of instances of $a$ in the training set;

2) all availabilities for couples with correspondent $c$ of instances of $a$ in the training set;

3) all availabilities for all couples of instances of $a$ in the training set;

4) all availabilities for the couple $(c, m)$ of instances of activity classes that can occur in the same place as $a$ can in the training set.

On the Orange4Home dataset, the number of availability values the occupant has to preset or choose from under each of those assumptions is respectively:
TABLE IV

DWRMSE AND ERROR RATE OF AVAILABILITY INFERENCE UNDER VARIOUS INDEPENDENCE ASSUMPTIONS

\begin{tabular}{lcc}
\hline & DWRMSE & Error rate \\
\hline Correspondent independence & 1.002 & 0.427 \\
Modality independence & 1.226 & 0.268 \\
Correspondent-and-modality independence & 1.393 & 0.489 \\
Activity independence & 0.637 & 0.151 \\
\hline
\end{tabular}

1) 20 activities $\times 11$ modalities $=220$;

2) 20 activities $\times 7$ correspondents $=140$;

3) 20 activities $=20$;

4) 8 places $\times 7$ correspondents $\times 11$ modalities $=616$.

which are all orders of magnitude smaller than the initial 1540 availability values.

Table IV presents the DWRMSE and average error rate of each of those 4 availability inference approaches based on independence assumptions, when the inferred availability is compared to the true availability value that was given under no assumption of independence. We can see that error rates and DWRMSE under any of those 4 assumptions are considerably bigger than when no such assumptions were made $(0.232$ DWRMSE and 0.015 error rate). In particular, both the correspondent independence assumption and the correspondentand-modality independence assumption have error rates nearing $50 \%$ ( 0.427 and 0.489 respectively), which is obviously unacceptable. Therefore, A1 and A3 are not valid assumptions.

Modality independence, despite having an error rate smaller than correspondent independence ( 0.268 against 0.427$)$, has a larger DWRMSE (1.226 against 1.002). This can be explained by the fact that activities that are the longest often induced specific preferences of modalities. For example in Orange4Home, computing in the office and napping in the bedroom are among the longest activities that occur: in both cases, the occupant has set their availability because of specific constraints (for example, they cannot receive synchronous communications during their sleep, and that they heavily favor emails during their work); under the modality independence assumption, those specificities are ignored and the errors done mostly occur on those long activities, thus penalizing the DWRMSE result. Therefore, A2 is not a valid assumption.

The activity independence assumption leads to smaller error rates and DWRMSE compared to the other 3 assumptions, although the performances are still heavily degraded compared to the no-assumption approach (0.637 against 0.232 DWRMSE and 0.151 against 0.015 error rate, see Tables III and IV). This rather limited decrease in performance suggests that places, ignoring activities performed in those places, are already a fairly important factor of influence over the availability of the occupant. Intuitively, this can be justified by the fact that places in the home are, in the Orange4Home dataset but also often in real life, single rooms with specific appliances and with a limited set of functions in the mind of the occupant. As such, activity classes that occur in the same place tend to be functionally linked (e.g. Cooking and 
TABLE V

Activity Instances Misclassified by The Place-Based MLP

\begin{tabular}{lllll}
\hline \multicolumn{2}{c}{ Ground truth } & & \multicolumn{2}{c}{ Decision } \\
\cline { 1 - 2 } Place & \multicolumn{1}{c}{ Class } & & Place & Class \\
\hline Bathroom & Cleaning & & Bedroom & Dressing \\
Bathroom & Using the sink & & Bathroom & Using the toilet \\
Bathroom & Using the sink & & Bathroom & Using the toilet \\
Entrance & Leaving & & Kitchen & Cleaning \\
Kitchen & Preparing & & Kitchen & Cleaning \\
Kitchen & Preparing & & Kitchen & Cleaning \\
Kitchen & Preparing & & Kitchen & Cleaning \\
Living room & Cleaning & & Kitchen & Cleaning \\
\hline
\end{tabular}

Washing the dishes in the Kitchen) and so the availability of the occupant is also often linked between those activities. Therefore, A4 can be a valid assumption, but will induce a significant drop in performances.

\section{E. Impact of Activity Recognition on Availability Estimation}

In the case where the activity class $a$ of an instance is the true class of the instance rather than the class decided by the place-based MLP, the inference approach of Section IV-C reaches a DWRMSE of 0.220 and an error rate of 0.007 . This error rate is approximately two times smaller than when recognized activity labels are used (0.015 in Table III). An F1 score gap of only $6.95 \%(100 \%-93.05 \%$, see Table II) during the activity recognition step thus causes a doubling error rate for the subsequent availability inference. In Table V, we report the 8 activity instances misclassified by the placebased MLP. We see that 5 of those instances were misclassified as an activity from the same place. Since place seems to be a good indicator of availability by itself (as shown in Section IV-D), the bias of our place-based approach, which tends to misclassify instances to classes of the same place, is beneficial in such systems.

\section{CONClusion}

From the conducted experiments presented in Section IV-C, we have seen that a simple averaging approach for inferring availability from a training set results in very small error rates (0.015) and DWRMSE (0.232). This indicates that a preferences-based approach, as was used in the Orange4Home data collection, can be used to accurately collect a training set that is representative of the desired availability of the occupant. In other words, an occupant will only sporadically change their availability from what they had preset.

As shown in Section IV-D, availability is heavily dependent on the correspondent, the modality as well as the activity of the occupant. Indeed, under various assumptions of independence, the performances of a baseline availability inference approach significantly worsen, which indicates that an occupant will not set their availability preferences identically regardless of the correspondent, regardless of the modality, regardless of both, or regardless of the activity.

However, we have observed that activities that occur in the same place have mostly similar availability preferences, so that misclassifying an instance as an activity that occurs in the same place as the true activity class is generally less harmful than completely misjudging the place in which the instance occurs. The place-based approach we have used in those experiments, when misclassifying instances, is biased towards activities of the same place, which is a desirable property for an activity recognition system used for availability inference.

\section{ACKNOWLEDGEMENT}

We thank Nicolas Bonnefond and Stan Borkowski for their help during the collection of the Orange4Home dataset. This work benefited from the support of the French State trough the Agence Nationale de la Recherche under the Future Investments program referenced ANR-11-EQPX-0002.

\section{REFERENCES}

[1] J. Cumin, G. Lefebvre, F. Ramparany, and J. L. Crowley, "A dataset of routine daily activities in an instrumented home," in $11^{\text {th }}$ International Conference on Ubiquitous Computing and Ambient Intelligence (UCAmI), 2017.

[2] L. D. Turner, S. M. Allen, and R. M. Whitaker, "Interruptibility prediction for ubiquitous systems: conventions and new directions from a growing field," in Proceedings of the 2015 ACM International Joint Conference on Pervasive and Ubiquitous Computing. ACM, 2015, pp. 801-812.

[3] T. Tanaka, R. Abe, K. Aoki, and K. Fujita, "Interruptibility estimation based on head motion and pc operation," International Journal of Human-Computer Interaction, vol. 31, no. 3, pp. 167-179, 2015.

[4] J. Fogarty, J. Lai, and J. Christensen, "Presence versus availability: the design and evaluation of a context-aware communication client," International Journal of Human-Computer Studies, vol. 61, no. 3, pp. 299-317, 2004.

[5] Y. Kobayashi, T. Tanaka, K. Aoki, and K. Fujita, "E-mail delivery mediation system based on user interruptibility," in International Conference on Human-Computer Interaction. Springer, 2015, pp. 370-380.

[6] Y. Takemae, T. Ohno, I. Yoda, and S. Ozawa, "Estimating human interruptibility in the home for remote communication," in CHI'O6 Extended Abstracts on Human Factors in Computing Systems. ACM, 2006, pp. 1397-1402.

[7] K. S. Nagel, J. M. Hudson, and G. D. Abowd, "Predictors of availability in home life context-mediated communication," in Proceedings of the 2004 ACM conference on Computer supported cooperative work. ACM, 2004, pp. 497-506.

[8] Y. Takemae, S. Chaki, T. Ohno, I. Yoda, and S. Ozawa, "Analysis of human interruptibility in the home environment," in CHI'07 Extended Abstracts on Human Factors in Computing Systems. ACM, 2007, pp. 2681-2686.

[9] J. Cumin, G. Lefebvre, F. Ramparany, and J. L. Crowley, "Human activity recognition using place-based decision fusion in smart homes," in International and Interdisciplinary Conference on Modeling and Using Context. Springer, Cham, 2017, pp. 137-150.

[10] W. G. Parrott, Emotions in social psychology: Essential readings. Psychology Press, 2001.

[11] K. S. Nagel, J.-Y. Sung, and G. D. Abowd, "Designing home availability services," Personal and Ubiquitous Computing, vol. 11, no. 5, pp. 361372, 2007.

[12] J. Fogarty, S. E. Hudson, C. G. Atkeson, D. Avrahami, J. Forlizzi, S. Kiesler, J. C. Lee, and J. Yang, "Predicting human interruptibility with sensors," ACM Transactions on Computer-Human Interaction (TOCHI), vol. 12, no. 1, pp. 119-146, 2005.

[13] J. Cumin and G. Lefebvre, "A priori data and a posteriori decision fusions for human action recognition," in 11th International Joint Conference on Computer Vision, Imaging and Computer Graphics Theory and Applications (VISAPP), 2016.

[14] M. Hall, E. Frank, G. Holmes, B. Pfahringer, P. Reutemann, and I. H. Witten, "The weka data mining software: an update," $A C M$ SIGKDD explorations newsletter, vol. 11, no. 1, pp. 10-18, 2009. 